\title{
Validation of a kinematic laserscanning system
}

\section{Harald Vennegeerts, Jens Martin, Matthias Becker and Hansjörg Kutterer}

\begin{abstract}
The increasing data rate of actual laser scanners qualifies these sensors for kinematic applications. In case of surveying static environments the scanner can be integrated into a moving platform system. Therefore two main tasks are outstanding. Position and orientation of the platform have to be registered throughout a freely driven trajectory. The second challenge is to synchronize the scanned spatial data with the time scale of the positioning unit. This paper presents the specification of a mobile mapping system using a phase-based laser scanner with a hybrid INS (Inertial Navigation System)/GPS solution. Among a description of the system structure it includes a software-based method to synchronize the scanned profiles with the trajectory. No extra hardware unit for time registration of scanned profiles is required. After all a spatial comparison of independently observed control points allows the assessment of the system performance.
\end{abstract}

Keywords. Mobile mapping, kinematic laserscanning, terrestrial lidar, synchronization.

\section{Introduction}

The distribution of terrestrial laser scanners (TLS) has increased in the last few years. Due to the now available level of automation, the number of applications is rising, too. On the hardware side these applications are limited by the range of distance measurement and shadowing effects. For this reason, scan positions may vary. Merging these positions is the task of registration. For this transformation task many investigations have been undertaken. One focus is the registration without the need of external sensors and artificial markers as described in Dold and Brenner (2006). The algorithms are based on good initial values and an environment existing of sufficient distinct features. In addition these procedures require a scan time, which increases proportionally with the number of scan positions. Elongated environments like streets can only be observed with high costs.

Local scan positions often have to be integrated into a reference system (georeferencing). For this purpose some scanned objects have to be referenced previously. External sensors for position and orientation can take over the task of transformation between scan positions. The drawbacks of the use of additional sensors are mainly costs and a restricted field of application, for example because of a limited availability of GPS. On the other hand, external sensors reduce the total scanning time, which is essential to establish and update mass data. For scanning large areas, for instance urban areas, kinematic laserscanning (airborne of terrestrial) is used. Several commercial solution can already be found, e.g. the StreetMapper project, which is described in Hunter et al. (2006). The accuracy of the scanned point cloud depends on the hardware used in the scanning process (positioning-, orientation- and scanning-unit). Further geometrical assessments can also be found in Barber et al. (2008).

In kinematic applications the relative spatial situation between scanner and object is changing with time. A common time scale is needed, which can be realized providing synchronized scanner and external sensors. Compared to the static case, the spatial transformation is thus extended by the time component. In the following the design of such a kinematic laserscanning system is described. This system includes different external sensors. The common time scale of positioning units and the scanner can be realized using different synchronization methods.

\section{Basic concept}

\subsection{Kinematic positioning}

Due to the fact that all scanned points have to be transformed into a unique reference system, positioning units have to refer to this absolute system too. For this reason local positioning units like total stations have to be orientated first, whereas GPS operates on a global system itself. In the present application the system should be capable of scanning a free trajectory driven on a street. Compared to smooth trajectories (e.g. rails), the positioning unit has to register high-frequency tilts and small radii of curvature. In addition the rate of recorded positions and orientations has to correspond to the high scan rate.

A hybrid INS/GPS solution fulfills these requirements. On the one hand, GPS provides a long-term accurate position, while lacking high update rates. Inertial navigation on the other hand can only sustain high accuracy for a short time due to the integration errors of angular velocities and linear accelerations, but with a high update rate for the calculated position and orientation, see Jekeli (2001). Apart from the integration of an IMU (Inertial Measurement Unit), orientation and position can also be supplied by a pure GPS solution for the trajectory, if the dynamics of the motion are moderate as in Hesse and Kutterer (2007).

Figure 1 gives an overview of the basic system concept. The system components are linked with spatial transformations and synchronizations, which are specified below. 


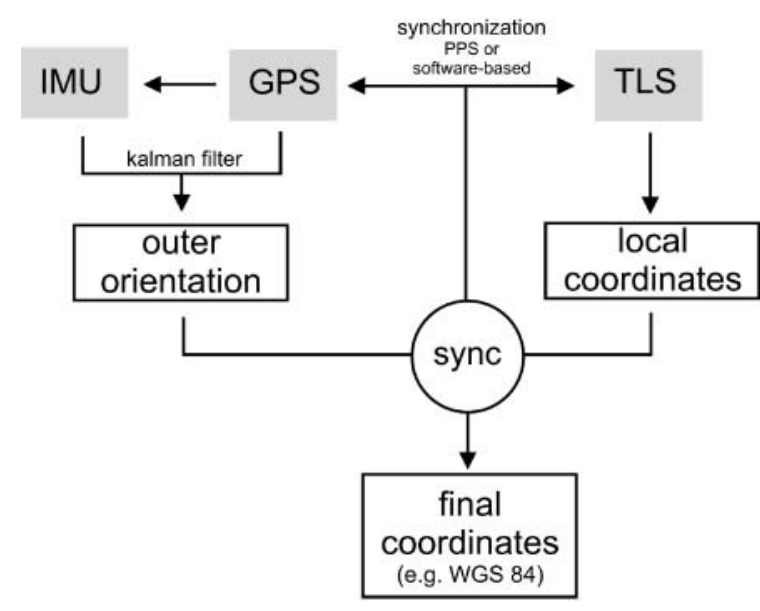

Figure 1: System overview.

\subsection{Spatial transformation}

One challenge for a hybrid measurement system is the spatial integration of all components. In this case, GPS, IMU and the laser scanner are independent units, using their own coordinate system:

- Laser scanning system

- Inertial (sensors) system of the IMU

- Body system, matching sensor system of IMU and GPS and TLS

- Navigation system (local level)

- Earth-Centered, Earth-Fixed (ECEF) system (e.g. World Geodetic System WGS84) for final coordinates.

Based on the laser scanner system, scanned points have to be transformed into the body system of INS/ GPS first. The parameters can be obtained by identical points, on the one hand determined in the body system (e.g. by close-range photogrammetry) and additionally scanned in the scanner system.

All points are registered in the local coordinate system of the laser scanner. The scanned profiles are time stamped to combine them with position and orientation provided by INS/GPS. A series of transformations is necessary to yield the scanned points in the final ECEF system.

$$
\mathbf{x}_{E}=\underbrace{\mathbf{R}_{N a}^{E} \underbrace{N a}_{\text {Coordinates in the ECEF system }} \underbrace{\left(\mathbf{R}_{S 3 D}^{B o} \mathbf{R}_{S 2 D}^{S 3 D} \cdot \Delta \mathbf{x}_{S 2 D}+\Delta \mathbf{x}_{S c}^{B o}\right)}_{\text {Coordinates in the body system }}}_{\text {Coordinates in the local level system }}+\Delta \mathbf{x}_{B o}^{E}
$$

The 2D coordinates of the points of each profile $\Delta \mathbf{x}_{S 2 D}$ are rotated into the $3 \mathrm{D}$ scanner system, according to the fixed azimuth orientation around the $\mathrm{Z}$-axis of the scanner $\mathbf{R}_{S 2 D}^{S 3 D}$. A six-parameter transformation (consisting of translation $\Delta \mathbf{x}_{S c}^{B O}$ and rotation $\mathbf{R}_{S 3 D}^{B o}$ ) leads to coordinates in the body system. The transformation to the navigation and ECEF system is provided by the Euler angles (DIN 9300) and the actual position. This position and orientation information is derived from a Kalman filter described below.

There are different possibilities combining GPS and INS measurements. The present application uses a loosely coupled Kalman filter, in which GPS and INS are considered independent systems, and are linked through the GPS position compared to the corresponding position calculated from the INS. A detailed description can be found in Heinze (1996). The principle of the Kalman filter is the recursive determination of the parameters (states) of the time discrete INS/GPS. It represents a dynamic system, based on a linear differential equation of first order.

$$
\dot{\vec{x}}_{(t)}=F_{(t)} \vec{x}_{(t)}+D_{(t)} \vec{w}_{(t)}
$$

with

$\vec{x}_{(t)} \quad$ state vector

$F_{(t)} \quad$ dynamic matrix

$D_{(t)}$ error matrix

$w_{(t)}$ stochastical error contribution.

The state vector contains the following elements:

$\varepsilon_{x}^{N}, \varepsilon_{y}^{N}, \varepsilon_{z}^{N} \quad$ misorientation [arcsec]

$\delta v_{x}^{N}, \delta v_{y}^{N}, \delta v_{z}^{N} \quad$ velocity error states $\left[\mathrm{ms}^{-1}\right]$

$\delta \lambda^{E}, \delta \varphi^{E}, \delta h^{E}$ position states [rad], [rad], [m]

$d_{x}^{B}, d_{y}^{B}, d_{z}^{B} \quad$ gyro drift stability [arcsec s ${ }^{-1}$ ]

$b_{x}^{B}, b_{y}^{B}, b_{z}^{B} \quad$ accelerometer drift stability $\left[\mathrm{ms}^{-1}\right]$.

The transition matrix for predicting the states is obtained by

$$
\phi(\Delta t)=I+F \Delta t+\frac{1}{2} F^{2} \Delta t^{2}+\cdots
$$

and for the time discrete case, the prediction (extrapolation) for each time step $\delta t$ results in

$$
\hat{\vec{x}}_{(t+1)}^{*}=\phi_{(t)} \hat{\vec{x}}_{(t)} \text {. }
$$

The update of the state vector after each measurement with position updates from GPS is computed by

$$
\hat{\vec{x}}_{(t+1)}=\hat{\vec{x}}_{(t+1)}^{*}+K_{(t+1)} \cdot\left(y_{(t+1)}-A \vec{x}_{(t+1)}^{*}\right)
$$

with the observation vector

$$
y_{(t+1)}=\left(\begin{array}{c}
\lambda_{(t+1)} \\
\phi_{(t+1)} \\
h_{(t+1)}
\end{array}\right)_{\mathrm{IMU}}-\left(\begin{array}{l}
\lambda_{(t+1)} \\
\phi_{(t+1)} \\
h_{(t+1)}
\end{array}\right)_{\mathrm{GPS}}
$$

and

A design matrix of the observables

$K_{(t)} \quad$ gain matrix of the filter process.

\section{Synchronization}

\subsection{Hardware module - Time reference with PPS}

From an external point of view, position and orientation of INS/GPS and laser scanner vary permanently 
as long as the platform is moving. All integrated sensors have to refer to a common time scale. On the part of INS/GPS, each IMU measurement has to be time stamped by an i/o card. The local timescale of the i/o card is updated every second with the GPS PPS (pulse per second) signal. The PPS signal can be used to time stamp the scanned profiles, too. We used a real-time processor and registered the PPS signal and the pulse of a scan profile on a uniform time scale. From the absolute time information of GPS, it is possible to determine the associated time in the GPS time scale for each profile. The latencies for the collection of the impulses and their flanks have to be considered.

The hardware-based time coding used in the allocation of the trajectory is compared in this paper to a software-based time synchronization. This alternative method is described in the following.

\subsection{Software-based approach to synchronize in object space}

An extra hardware unit for the registration of the scanned profiles has to meet the demands of the high scan rate. For this reason a software-based method is developed to synchronize laser scanner and INS/ GPS. The procedure uses the rotating mirror of the scanner as a clock. Previous investigations found in Schulz (2007) indicate the stability of angular velocity during the scan. Assuming a constant rotation velocity, the two parameters time shift and drift can be estimated based on geometrical conditions.

The procedure aims at setting time shift and drift regarding to a reference time scale, which exists due to the integration of GPS. The only necessary condition for the synchronization is to scan very few control points with known position. These points serve as reference points in time (hereinafter called sync points). The preferred position of these sync points is at the beginning and at the end of the trajectory. They can be either measured with an external GPSReceiver (e.g. RTK) or - more suitably - with a 3D scan at the beginning during the initialization phase of the INS.

With the known relative orientation of IMU and scanner, a single profile is first approximated by a plane (Figure 2). The IMU determines the orientation of each plane along the trip. Figure 3 shows the distance $d_{p}$ of every sync point to the planes in relation to the time of a straight trajectory.

Analytically, the orthogonal distance can be expressed as a function $d_{p}=f\left(t_{i}\right)$ for a sync point at time $t_{i}$. The minimum of this function corresponds to the time, when the scanner registers the sync point regarding to geometrical conditions.

$$
t_{p}=f^{-1}\left(\min \left(d_{p}\right)\right)-[\phi / 2 \pi \cdot \omega]
$$

The additional term indicates that the scan time determined by an approximated plane is always too

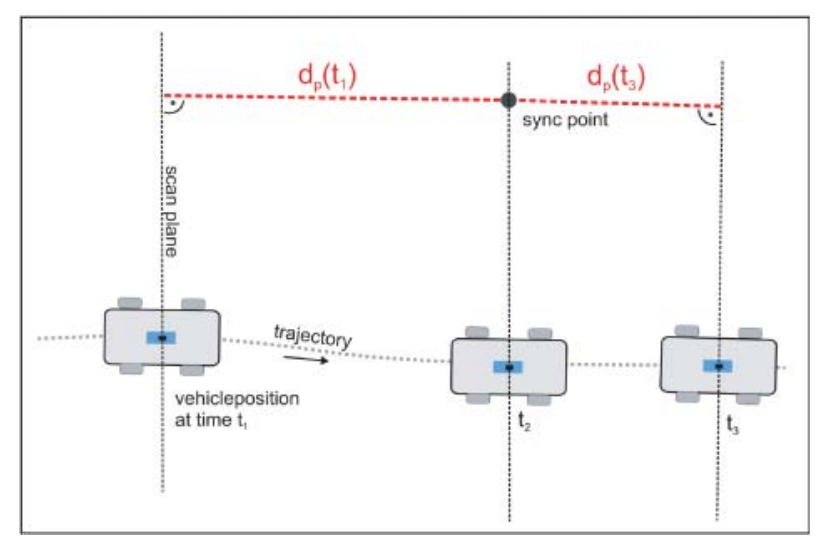

Figure 2: Orthogonal plane distances at different vehicle positions.

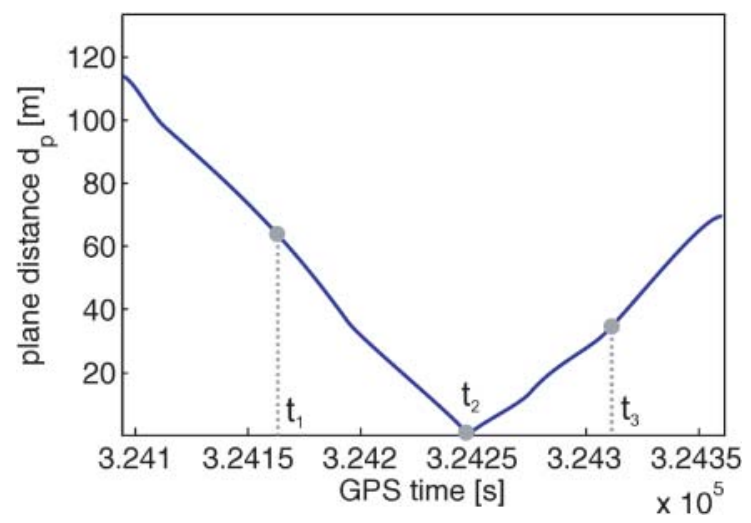

Figure 3: Orthogonal distance of approximated scan planes to sync points.

late. This is caused by the fact that the vehicle is moving during scan, which results in a helix structure of the scan path. The time of the minimum distance refers to the scan time of a single point instead of the characteristic profile time, when the mirror passes the nadir. This time delay is considered using the vertical angle $\phi$ relative to the angular velocity $\omega$. Figure 3 shows the orthogonal distances at discrete high frequently generated times.

On the other hand the intensity image of the scan provides the profile, the sync point has been scanned in fact. The crucial value is the first profile, the sync point appears. This profile number $n_{i}$ of the scan image can be used to generate a time scale $t_{s}$ based on the rotation velocity $\omega$ of the mirror starting at $t_{0}$.

$$
t_{s}=n_{i} \cdot \omega+t_{0}
$$

With unknown rotation velocity at least two points are necessary to set shift and drift or rotation velocity, respectively. In case of using more than two sync points, the parameters can be estimated by a simple regression analysis. The result of the estimated relation between the time scales can be seen in Figure 4. 


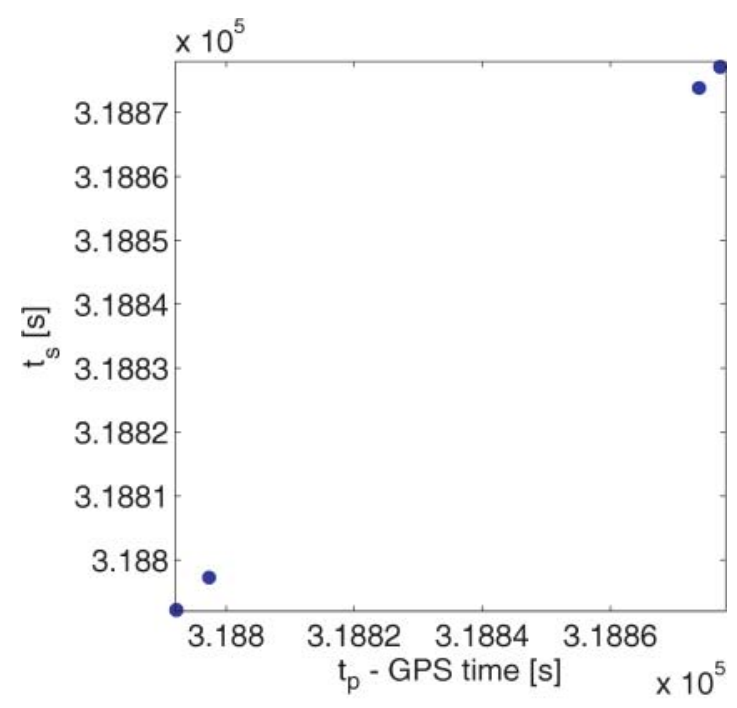

Figure 4: Estimated relation between the time scales $\left(t_{p}\right.$ generated out of geometrical conditions and $t_{s}$ out of appearance in scan image). The scan times between the capture of the sync points are bridged on the assumption that the mirror rotates constantly (see Figure 6).

Table 1: Comparison of rotation time (scan mode $33 \mathrm{rps}$ ) using a scanner identical in construction, once estimated by synchronization in object space and again by laboratory tests as in Schulz (2007).

\begin{tabular}{llll}
\hline & \multirow{2}{*}{$\begin{array}{l}\text { sync in } \\
\text { object space }\end{array}$} & \multicolumn{2}{l}{ laboratory test } \\
\cline { 3 - 4 } & setup 1 & setup 2 \\
\hline Rotation time $[\mathrm{s}]$ & 0.030547 & 0.030543 & 0.030545 \\
\hline
\end{tabular}

Table 1 shows the results for a drift parameter, exemplarily estimated from a test run. If a long-term drift can be excluded by using an external PPS signal, the parameter can be interpreted as the rotation velocity of the vertically rotating mirror. The rotation time of the test run corresponds to previous laboratory tests as in Schulz (2007) with a deviation of $10^{-6} \mathrm{~s}$.

The requirements regarding the precision of an estimated rotation velocity are related to the data rate, the total time of scanning, the velocity of the vehicle and the required spatial accuracy.

Assuming the constant rotation period is known with a precision of $1 \mu \mathrm{s}$, a resulting deviation in space depends on the velocity of the vehicle and on the total time of scanning. For example a scanning sequence of five minutes (10000 profiles at $33 \mathrm{rps}$ ) with an absolute known rotation velocity of $1 \mu$ s leads to an accumulated deviation of $10 \mathrm{~ms}$ at the end of the sequence. A scanning vehicle driven with $2 \frac{\mathrm{m}}{\mathrm{s}}$ causes a spatial deviation of $2 \mathrm{~cm}$. Vice versa the parameter precision of sync points, scanner position and orientation as well as the vehicle velocity influence the estimation of an unknown rotation velocity.
Table 2: Technical specification of the inertial measurement unit "Honeywell Lasernav II".

\begin{tabular}{ll}
\hline Measuring rate & $50 \mathrm{~Hz}$ \\
Gyro resolution & $0.001^{\circ}$ \\
Gyro bias & $0.01 \frac{\circ}{\mathrm{hr}}$ \\
Gyro scale factor & $20 \mathrm{ppm}$ \\
Acc bias stability & $0.06 \mathrm{mg}$ \\
Acc scale factor & $5 \mathrm{ppm}$ \\
\hline
\end{tabular}

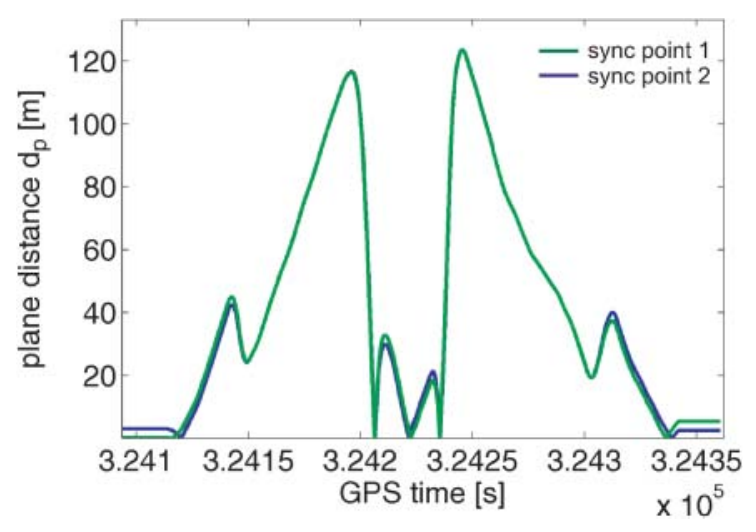

Figure 5: Orthogonal distances $d_{p}$ of two sync points, situated at the begin and at the end of an oval trajectory.

\section{Validation}

For our investigations we used a INS/GPS consisting of a geodetic GPS Receiver and a laser gyro IMU "Honeywell Lasernav II". The inertial system provides position and orientation data with a rate of $50 \mathrm{~Hz}$. Table 2 contains the technical details of the IMU.

In addition to the INS/GPS we integrated a phasedbased laser scanner (Leica HDS 4500), set to 2D profile mode. The scanner allows a vertical rotation rate of $33 \mathrm{rps}$. To achieve a spatial resolution of about $6 \mathrm{~cm}$ in driving direction, we chose a speed limit of the mobile system of about $2 \frac{\mathrm{m}}{\mathrm{s}}$. This spatial resolution is necessary to recognize control points for validation. The result of the test drives are threedimensional consistent and geo-referenced point clouds calculated by equation 1 . With two different test drives the potential of the software-based synchronization (Section 4.1) and an absolute spatial comparison (Section 4.2) are demonstrated in the following subsections.

\subsection{Potential of software-based synchronization}

We tested the software based synchronization in several practical case studies. For an oval trajectory two sync points were scanned during the initialization phase of the IMU. These two points were scanned twice, at the beginning and at the end of the test drive. The shape of the trajectory and the position of the sync points leads to the time-distance diagram of Figure 5. 


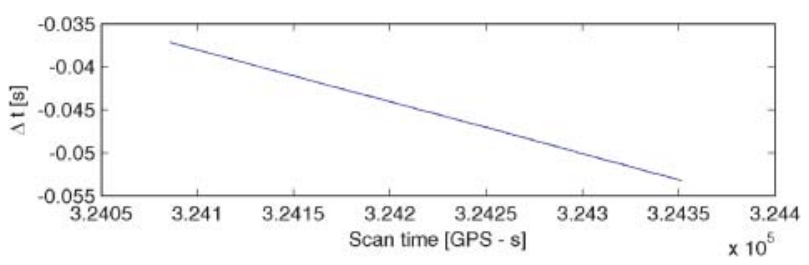

Figure 6: Compared profile times from profile impulse times (registered hardware-based) minus object space times (registered software-based).

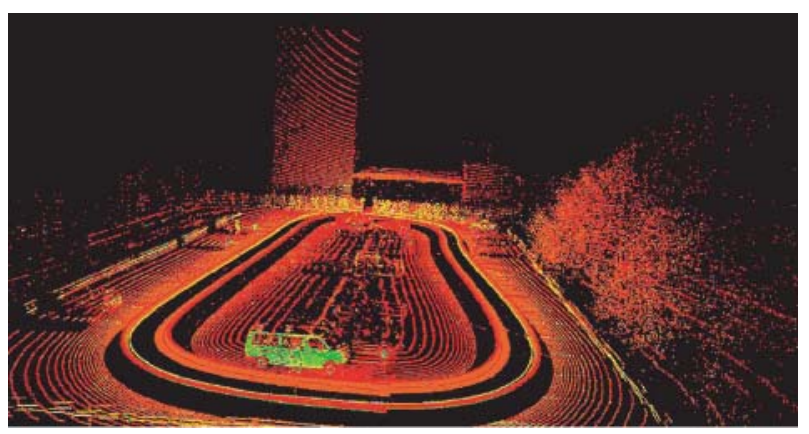

Figure 7: Point cloud (times software-based synchronized).

In this trajectory there exist five possible candidates for a minimum orthogonal distance $d_{P}$. The ambiguity can be solved with the information, that each sync point was registered twice. Together with the scan image we estimated the profile times. Altogether, in the scan image there is one profile less than the number of impulses registered. The compared profile times (Figure 6) indicate that the first profile impulse has to be cut off. The maximum remaining differences $(|\Delta t|<0.025 \mathrm{~s})$ result from the adjusted parameter of the mirror rotation period. The linearity of the differences confirms a constant rotation velocity.

On the condition, that the sync points can be identified uniquely in the intensity image and the rotation velocity of the mirror is constant, the synchronization does not depend on driving speed and driving time. The performance of the positioning unit and the variance of the sync points limit the potential of the software-based synchronization. The synchronization is therefore as precise as the orthogonal distances $d_{p}$ can be estimated. So we used the sync points to interpolate the profiles in driving direction.

\subsection{Spatial comparison to absolute control points}

This case study shows a comparison between the spatial control points measured independently by RTKGPS on the one hand and their coordinates calculated from the GPS/INS/TLS measurement system during the drive on the other hand.

The control points were located at the beginning and in the middle of the trajectory.

Figure 7 shows exemplarily a point cloud scanned during an oval test drive. The time synchronization
Table 3: Results of the spatial investigation: Cartesian differences of control point coordinates, measured by RTK-GPS and the kinematic laserscanning system.

\begin{tabular}{rrrr}
\hline Point & \multicolumn{3}{c}{ Differences Body system $[\mathrm{mm}]$} \\
\cline { 2 - 4 } & $\mathrm{X}$ & $\mathrm{Y}$ & \multicolumn{1}{c}{$\mathrm{Z}$} \\
\hline 169 & 11 & 33 & 36 \\
166 & 18 & 40 & 12 \\
165 & -2 & 33 & 3 \\
341 & 21 & -5 & -2 \\
1000 & 44 & -48 & -24 \\
\hline
\end{tabular}

between INS/GPS and laser scanner was done using the software based approach (see Section 3.2). The differences to single control points (Table 3 ) allow to demonstrate the superior performance of the system. Note, that there are no systematic errors due to the software based synchronization, which would result in a translation on the $\mathrm{Y}$-axis expressed in body system coordinates.

The difference between control points and measured points expressed in the body system identifies potential error sources, because the body system is a righthanded system oriented as follows:

- $\mathrm{X}$-axis points to the right

- Y-axis points to the direction of motion

- Z-axis point upwards.

Errors in time synchronization between the laser scanner and the GPS/IMU would yield a systematic offset in the direction of motion. Misorientation around the Z-axis could also be detected by placing control points on both sides of the trajectory. For instance a positive error in the $\mathrm{Z}$-axis rotation would result in positive body system $\mathrm{Y}$-axis differences on the right side of the trajectory and negative body system $\mathrm{Y}$-axis differences on the left side. As shown in Table 3, no significant errors were detected.

According to the typical error budget of GPS positioning and the spatial resolution of the profiles in driving direction, the differences are within an expected range.

Furthermore, to check synchronization and positioning, we proved the flatness of a neighboring wall, scanned while passing through a narrow curve on a parking ground. Figure 8 illustrates the orthogonal residuals to a plane, fitted to the scanned points. As illustrated by the color code, no systematic effects are visible. Because the wall was scanned in curve, a synchronization error would result in a curved surface of the flat wall.

\section{Conclusions and outlook}

The integration of information from external sensors like INS/GPS for terrestrial laser scanning allows time-saving scans with an absolute uncertainty of few centimeters. The drawbacks of the systems are 


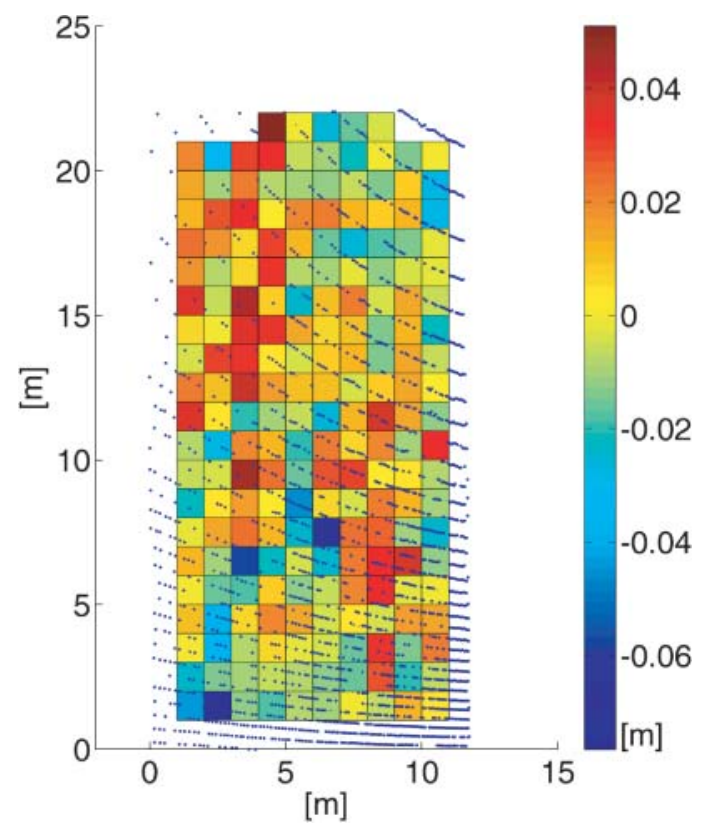

Figure 8: Flatness of a scanned wall. Colors represent the deviation from a plane.

high costs, required robustness of the positioning unit (especially sufficient GPS coverage) and the additional task of sensor sychronization. We propose an approach, which considers the constant rotation velocity of the vertical mirror. We integrate information from object space for the task of synchronization and extend the usual one way analysis using only sensor data but not including geometrical conditions.

Regarding hardware, future work will include the use of low cost sensors, e.g. antenna array receiver based on GPS L1 or low cost IMU as in Hwang et al. (2005). Side-viewing scanning is certainly suitable to capture laminar spatial data like building facades. However, objects outside the field of view (e.g. interior edges) are shadowed. This limitation can be overcome using multiple or rotating sensors. Such configurations provide redundant data, which can be used for a refined analysis.

Similar to automatic registration methods for static scans, information from object space bears the potentials for positioning tasks. In addition to hardware developments, software-based approaches can improve the effectiveness and interpretation of scanned spatial data.

\section{References}

Barber, D., Mills, J., and Smith Voysey, S., Geometric validation of a ground-based mobile laser scanning system, ISPRS Journal of Photogrammetry and Remote Sensing 63:1 (2008), 128-141.

Dold, C. and Brenner, C., Registration of Terrestrial Laser Scanning Data using Planar Patches and Image Data, Proceedings of the ISPRS Commission V Symposium 'Image Engineering and Vision Metrology' (2006), 78-83.

Heinze, O., Aufbau eines operablen inertialen Vermessungssystems zur Online-Verarbeitung in der Geodäsie auf Basis eines kommerziellen Strapdown Inertialsystems, $\mathrm{PhD}$ thesis, Technische Universität Darmstadt, Institut für Physikalische Geodäsie, 1996.

Hesse, C. and Kutterer, H., A mobile mapping system using kinematic terrestrial laser scanning (KTLS) for image acquisition, Proceedings on the VIII Optical 3D Measurement Techniques, Zürich (2007).

Hunter, G., Cox, C., and Kremer, J., Development of a Commercial Laser Scanning Mobile Mapping System - StreetMapper, Second International Workshop The Future of Remote Sensing, Antwerp (2006).

Hwang, D.-H., Oh, S. H., Lee, S. J., Park, C., and Rizos, C., Design of a low-cost attitude determination GPS/INS integrated navigation system, GPS Solutions 9 (2005), 294-311.

Jekeli, C., Inertial Navigation Systems with Geodetic Applications, Walter de Gruyter, 2001.

Schulz, T., Calibration of a Terrestrial Laser Scanner for Engineering Geodesy, Diss., Eidgenössische Technische Hochschule ETH Zürich, Nr. 17036, 2007.

Received: Nov 29, 2007

Accepted: Jan 31, 2008

\author{
Author information \\ Harald Vennegeerts and Hansjörg Kutterer \\ Geodetic Institute \\ Gottfried Wilhelm Leibniz Universität Hannover \\ Nienburger Str. 1 \\ 30167 Hannover, Germany \\ E-mail: vennegeerts@gih.uni-hannover.de, \\ kutterer@gih.uni-hannover.de \\ Jens Martin and Matthias Becker \\ Institute of Physical Geodesy \\ Technische Universität Darmstadt \\ Petersenstr. 13 \\ 64287 Darmstadt, Germany \\ E-mail: martin@ipg.tu-darmstadt.de, \\ becker@ipg.tu-darmstadt.de
}

